\title{
Recién nacido de muy bajo peso de nacimiento; análisis de mortalidad
}

\author{
Dr. L. Barranco Córdoba, * \\ Dra. L. Kyling Montecinos**
}

\begin{abstract}
A retrospective study of 35 Chnical records of new born infants, bom between dugust 15t), 79 and August 30th., 80 , with a birth-weight of $1.500 \mathrm{gr}$ or less, was made.

The incidence of weight, gestational age and Apgar score upon mortality, was analized. $54,2 \%$

The incilence of very low weight new borns was $1.35 \%$ and the rate of mortality of this specitic gromp was
\end{abstract}

En la actualidad, los cuidados intensivos en los Recién Nacidos (R.N.) de muy bajo peso de nacimiento (MBPN), han logrado reducir tanto los niveles de mortalidad (a 15-24\% en el grupo de 1.000 a 1.500 gramos al nacer $y$ a $50-70 \%$ para los menores de $1 .(000 \text { gramos) })^{1}$ como las secuelas por daño neurológico. ${ }^{2}$

"Jefe Unidad de Nennatología, Imstituto de Pediatría, Un niversidad Austral de Chile.

** Médicu en Especialidaxd Básica en Estada de Perfeccionimiento, Servicio Pediatria, Hospital Regional de Valdivia

Trabajo incorporación a Sociedad Chilena de Pediatrias, Presentudo en VIII Jomadas Nacionales de Pediatria, diciembre 1960. Arica.
A pesar de que el cuidado intensivo se inicia a nivel mundial en $1960^{1}$ en nuestro país sólo en $1976^{3}$ se planifica una implementación nacional, que se está haciendo efectiva en algunos lugares desde el año 1979 en adelante.

Al presentar este grupo de Recién Nacidos de MBPN pretendemos establecer, con algunos parámetros, cuál es la situación en la Unidad de Recién Nacidos del Hospital Regional de Valdivia, en donde esperamos que los cuidados intensivos entren a corto plazo en funcionamiento en fonma completa.

Este estudio se propone:

Relacionar la mortalidad de los R.N. de MBPN 
con los parámetros de: peso, edad gestacional (E.G.) y Apgar al nacer.

Analizar la incidencia de la asfixia neonatal (NN) en relación con el peso y la edad gestional en este grupo.

\section{MATERIAL Y METODO}

Revisión retrospectiva de fichas clínicas de 35 R.N., que pesaron 1.500 gr o menos al nacer, hospitalizados en la Unidad de Neonatología en Hospital Regional de Valdivia, desde el 15 de agosto de 1979 al 30 de agosto de 1980 .

Los 35 niños se dividieron en 3 grupos de acuerdo a su peso: de $1.000 \mathrm{gr}$ o menos, de 1.001 a 1,200 gr y de 1.201 a $\mathrm{I} .500 \mathrm{gr}$.

La Edad Gestacional se estableció según método de Usher ${ }^{4}$ y el examen neurológico adaptado de Amiel Tisson., ${ }^{5}$

El puntaje de Apgar se tomó al primer minuto y a los 5 minutos, separando 3 grupos: Apgar 0-3, asfixia N.N. severa; Apgar 46 como asfixia N.N. moderada; registro incompleto al minuto (2 niños) $y$ a los 5 minutos ( 10 niños).

\section{RESULTADOS}

De los 35 R.N. estudiados; el $22,9 \%$ pes 6 al nacer 1.000 gr o menos y el $62,9 \%$ entre 1.201 y 1.500 gramos (Tabla 1).

Las edades gestacionales fuctuaron entre $24 \mathrm{y}$ 35 semanas (Tabla 2). En esta distrilucción el $71,2 \%$ tenía 30 semanas o menos y el $22,7 \%$ menos de 28 semanas de edad gestacional.
Según Apgar (Tabla 3), el 66\% de estos nin̄os presentan algún grado de asfixia N.N. al minuto de vida, que en el $\mathbf{4 0 \%}$ erd severa. Estas cifras se reducen a la mitad, al considerar el Apgar a los 5 minutos, persistiendo un $14 \%$ de ellos en depresión severn.

Tabla 1

Peso

Recién nacidos de MBPN

Hospital Regional de Valdivia

\begin{tabular}{|c|c|c|}
\hline PESO EN GR & N. ${ }^{\circ}$ DE CASOS & PORCENTAJE \\
\hline $500-1000$ & 8 & 22.9 \\
$1001-1200$ & 5 & 14.2 \\
$1201-1500$ & 22 & 62.9 \\
\hline
\end{tabular}

Tabla 2

Edad gestacional (Usher-Amiel Tison)

Recién nacidos de MBPN

Hospital Regional de Valdivia

\begin{tabular}{|c|c|c|}
\hline SEMANAS & N. ${ }^{\circ}$ DE CASOS & PORCENTAJE \\
\hline 22 & 4 & 811 \\
\hline 25 & 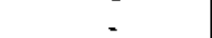 & $V / 1$ \\
\hline 26 & 1 & V1/, \\
\hline 27 & 3 & 111 \\
\hline 28 & 7 & $8 / 1, \quad 20$ \\
\hline 29 & 3 & 8,57 \\
\hline 30 & 7 & 20 \\
\hline 31 & 4 & 11,4 \\
\hline 32 & 4 & 11,4 \\
\hline 33 & I & 2,8 \\
\hline 34 & - & . \\
\hline 35 & $\mathbf{l}$ & 2,8 \\
\hline
\end{tabular}

$Z \bar{Z}=712 \%$.

Tabla 3

Apgar

Recién nacidos de MBPN

Hospital Regional de Valdivia

\begin{tabular}{|l|c|c|r|r|r|r|r|}
\hline APGAR & \multicolumn{2}{|c|}{$0-3$} & \multicolumn{2}{c|}{$4-6$} & \multicolumn{2}{c|}{$7-10$} & \multicolumn{2}{c|}{ NO CONSIGNADO } \\
\hline Tpo. en minutos & $1^{\prime}$ & 5 & 1 & 5 & 1 & 5 & 1 \\
\hline N."de casos & 14 & 5 & 9 & 7 & 8 & 13 & 2 \\
Porcentaje & 40 & 14 & 26 & 20 & 13 & 37 & 10 \\
\hline
\end{tabular}


Esta Unidad de Neonatología es un Centro de referencia de todos los hospitales de la Provincia de Valdivia donde el $14,3 \%$ de los R.N. de MBPN provienen de un Area rural. (Tabla 4).

Tabla 4

\section{Procedencia}

Recién nacidos de MBPN Hospital Regional de Valdivia

\begin{tabular}{|l|c|c|}
\hline & N.O DE CASOS & PORCENTAJE \\
\hline Area & $\mathbf{5}$ & 14.3 \\
Valdivia & 30 & 85 \\
\hline
\end{tabular}

En relación a la paridad materna, no existen diferencias significativas entre la madre inultipara y primípara.

Tabla 5

Paridad materna

Recién nacidos de MBPN Hospital Regional de Valdivia

\begin{tabular}{|l|c|c|}
\hline & N. DE CASOS & PORCENTAJE \\
\hline Primípara & 14 & 40,5 \\
Multípara & 14 & 40,5 \\
Gran multípara & 1 & 1 \\
\hline
\end{tabular}

En relación del tipo de parto (Tabla 6 ); el $59 \%$ cortespondió a parto eutócico, el $21,8 \%$ a cesárea y el $18,75 \%$ a partos en presentación podálica.

Tabla 6

Tipode parto

Reçién nacidos de MBPN Hospital Regional de Valdivia

\begin{tabular}{|l|c|c|}
\hline & N. ${ }^{\circ}$ DE CASOS & PORCENTAJE \\
\hline Eutócico & 19 & 59,3 \\
Cesárea & 7 & 21,8 \\
Podálica & 6 & 18,75 \\
\hline
\end{tabular}

La mortalidad global fue de un $54,2 \%$ y de los nacidos en Hospital Regional Valdivia falleció el $56,7 \%$ (Tabla 7$)$.

Al relacionar el peso con la mortalidad, se observa que fallecieron todos los R.N. con peso de 1.000 gramos o menos, El mayor peso de nacimiento se acompaña de mayor posibilidad de sobrevida, siendo èsta de $63,6 \%$ en los mayores de 1.201 gr. (Tabla 8 .)

Al relacionar la edad gestacional con la mortalidad (Tabla 9) se observa que, a menor edad gestacional, menor probabilidad de sobrevida: fallecen

Tabla 7

Mortalidad - Procedencia

Recién nacidos de MBPN Hospital Regional de Yaldjvia

\begin{tabular}{|l|c|c|c|c|}
\hline & \multicolumn{2}{|c|}{ vIVOS } & \multicolumn{2}{c|}{ FALLECIDOS } \\
\hline & N..$^{0}$ DE CASOS & PORCENTAJE & N. ${ }^{\circ}$ DE CASOS & PORCENTAJE \\
\hline Area & 3 & 60 & 2 & 40 \\
Valdivia & 13 & 43,3 & 17 & 56,7 \\
Total & 16 & 45,8 & 19 & 54,2 \\
\hline
\end{tabular}

Tabla 8

Mortalidad - Peso

Recién nacidos de MBPN Hospital Regional de Valdivia

\begin{tabular}{|c|c|c|c|c|}
\hline \multicolumn{2}{|c|}{ VIVOS } & \multicolumn{2}{c|}{ FALLECIDOS } \\
\hline PESO EN GR & N. $^{\circ}$ DE CASOS & PORCENTAJE & N. ${ }^{\circ}$ DE CASOS & PORCENTAJE \\
\hline $500-1000$ & - & - & 8 & 100 \\
$1001-1200$ & 2 & 40 & 3 & 60 \\
$1201-1500$ & 14 & 63,6 & 8 & 36,7 \\
\hline
\end{tabular}


Tabla 9

Mortalidad - Edad gestacional

Recién nacidos de MBPN

\begin{tabular}{|c|c|c|c|c|}
\hline \multirow[b]{2}{*}{$\begin{array}{l}\text { SEMANA } \\
\text { CESTACION }\end{array}$} & \multicolumn{2}{|c|}{ VIVOS } & \multicolumn{2}{|c|}{ FALLECIDOS } \\
\hline & N. DE CASOS & PORCENTAJE & N. ${ }^{\circ}$ DE CASOS & PORCENTAJE \\
\hline $\begin{array}{l}24 \\
25 \\
26 \\
27 \\
28 \\
29 \\
30 \\
31 \\
32 \\
33 \\
34 \\
35\end{array}$ & $\begin{array}{l}- \\
- \\
- \\
- \\
3 \\
3 \\
2 \\
3 \\
3 \\
1 \\
- \\
-\end{array}$ & $\begin{array}{c}- \\
- \\
- \\
- \\
42,8 \\
42,8 \\
- \\
42,8 \\
42,8 \\
- \\
- \\
-\end{array}$ & $\begin{array}{l}4 \\
- \\
1 \\
3 \\
4 \\
- \\
5 \\
1 \\
1 \\
- \\
- \\
-\end{array}$ & 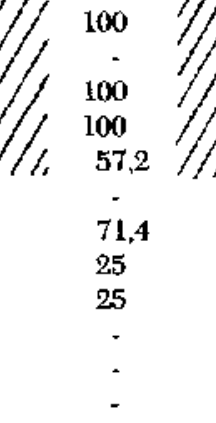 \\
\hline
\end{tabular}

$\mathbb{Z}=63,1 \%$

Tabla 10

Mortalidad - Apgar al minuto

Recién nacidos de MBPN

\begin{tabular}{|c|c|c|c|c|}
\hline & \multicolumn{2}{|c|}{ VIVOS } & \multicolumn{2}{c|}{ FALLECIDOS } \\
\hline & N." DE CASOS & PORCENTAJE & N. ${ }^{\circ}$ DE CASOS & PORCENTAJE \\
\hline $0-3$ & $\mathbf{5}$ & 35,7 & 9 & 64,2 \\
$4 \cdot 6$ & $\mathbf{5}$ & $\mathbf{4 5 , 4}$ & 6 & $\mathbf{5 4 , 5}$ \\
$7-10$ & 6 & - & 3 & 33,3 \\
\hline
\end{tabular}

Tabla 11

Apgar - Peso

Recién nacidos de MBPN

\begin{tabular}{|c|c|c|c|c|c|c|c|}
\hline \multirow{2}{*}{ APGAR } & \multirow{2}{*}{$\begin{array}{c}\text { TIEMPO } \\
\text { EN MINUTO }\end{array}$} & \multicolumn{2}{|c|}{$500-1000$} & \multicolumn{2}{|c|}{$1001-1200$} & \multicolumn{2}{|c|}{$1201-1500$} \\
\hline & & N." & $\%$ & N. ${ }^{n}$ & $\%$ & N. ${ }^{\circ}$ & $\%$ \\
\hline $0-3$ & $\begin{array}{l}1 \\
5\end{array}$ & $\begin{array}{l}7 \\
5\end{array}$ & 100 & - & - & $\begin{array}{l}7 \\
1\end{array}$ & 31,8 \\
\hline $4-6$ & $\begin{array}{l}1 \\
5\end{array}$ & - & 16,6 & $\begin{array}{l}2 \\
1\end{array}$ & 40 & 9 & 40,9 \\
\hline $7 \cdot 10$ & $\begin{array}{l}1 \\
5\end{array}$ & - & - & $\begin{array}{l}3 \\
2\end{array}$ & 60 & $\begin{array}{r}6 \\
11\end{array}$ & $\begin{array}{l}27,2 \\
64,2\end{array}$ \\
\hline
\end{tabular}

63,1\% de los que tienen 28 semanas o menos, $y$ sobreviven $63,1 \%$ de los que tienen 29 semanaso más.

La incidencia de la asfixia N.N. sobre la mortalidad se puede observar en la tabla 10 , en la cual $64,2 \%$ de los que tienen asfixia severa fallecen; mueren $54,5 \%$ de los que presentan asfixia nroderada y en forma global $83,3 \%$ de los que fallecen presentan algún grado de asfixia. Por otro lado, de los sobrevivientes $62,5 \%$ presentan algún grado de asfixia.

Al analizar las condiciones de vitalidad (Apgar) 
al nacer de los R.N. y relacionándolas con el peso, se observa (Tabla 11 ) que aquellos niños que pesan 1.000 gramos o menos al nacer presentan en el $100 \%$ de los casos asfixia severa y de éstos un porcentaje bajo $(16,6 \%)$ logra recuperarse parcialmente. Entre 1.001 y 1.200 gramos de peso, la asfixia disminuye a un $40 \%$. Entue 1.20 l y 1.500 gramos al nacer, llama la atención que un gran porcentaje de estos niños presentan algún grado de asfixia, pero el $64,2 \%$ logra una recuperación completa a los 5 minutos.

Situación similar se aprecia al hacer la relación entre Apgar $y$ edad gestacional (Tabla 12), que muestra que el $100 \%$ de los R.N. con menos de 28 semanas de gestación nacen severamente deprimidos, y a medida que la E.G. atumenta disminuye la incidencia de asfixia N.N.

Tabla 12

Apgar - Edad gestacional

Fecién nacidos de MBPN

\begin{tabular}{|c|c|c|c|c|c|c|}
\hline & & $\mathbf{A}$ & $G$ & A & & \\
\hline & \multicolumn{2}{|c|}{$0-3$} & \multicolumn{2}{|c|}{$4-6$} & \multicolumn{2}{|c|}{$7-10$} \\
\hline $\begin{array}{l}\text { EDAD } \\
\text { CESTACIONAL }\end{array}$ & $\begin{array}{l}\text { N.'DE } \\
\text { CASOS }\end{array}$ & $\begin{array}{l}\text { PORCEN- } \\
\text { TAJE }\end{array}$ & $\begin{array}{l}\text { N.'DE: } \\
\text { CASOS }\end{array}$ & $\begin{array}{l}\text { PORCEN- } \\
\text { TAJE }\end{array}$ & $\begin{array}{l}\text { N.DE } \\
\text { CASOS }\end{array}$ & $\begin{array}{l}\text { POHCEN- } \\
\text { TAJE }\end{array}$ \\
\hline 24 & 4 & 100 & - & . & - & - \\
\hline 25 & - & - & - & - & - & - \\
\hline 26 & 1 & $I(x)$ & - & - & - & - \\
\hline 27 & 2 & 100 & - , & - & - & - \\
\hline 28 & 3 & 42,8 & 3 & 42,8 & 1 & 14,2 \\
\hline 29 & 1 & 33,3 & - & - & 2 & 66,6 \\
\hline 30 & - & & 5 & 71,4 & 2 & 28,5 \\
\hline 31 & 2 & 50 & I & 25 & 1 & 25 \\
\hline 32 & $\mathbf{l}$ & 25 & 1 & 25 & 2 & 50 \\
\hline 33 & - & - & 1 & 100 & - & - \\
\hline 34 & - & - & - & - & - & - \\
\hline 35 & - & - & - & - & 1 & 100 \\
\hline
\end{tabular}

\section{DISCUSION X COMENTARIO}

La tendencia actual es continuar denominando niños de "bajo peso de nacimiento" a todo R.N. que pesa 2.500 gramos o menos al nacer, Sin embargo, con los avances experimentados por la neonatología, se ha introducido un nuevo término el de "muy bajo peso" (MPBN) para todo aquel F.N. que pesa menos de 1.500 gramos al nacer. A su vez se está tendiendo a dividir a estos niños en 3 grupos de acuerdo a su peso de nacimiento: menores o iguales a 1.000 gramos, 1.001 a $1.200 \mathrm{gra}-$ mos y de 1.201 a 1.500 gramos. $^{6}$

Al analizar los 35 R.N. presentados, podemos concluir que tanto el peso como la edad gestacional son parámetros que están relacionados directamente con la incidencia de Hipoxia perinatal y con la mortalidad?

Los promedios de Apgar presentados por el grupo estudiado fueron de 4,5 al minuto y 5,5 a los
5 minutos. En estudios efectuados en R.N. de 1.500 gramos o menos ${ }^{7}$ se observaron cifras de 3.3 al minuto y 4.8 a los 5 minutos, demostrando la alta incidencia de asfixia en R.N. de M B P N; y que a menos peso y menor E.G. mayor es la incidencia de asfixia N.N. De aquí que las acciones que actualmente se realizan en estos niños tiendan a lograr una sobrevida sin daño tísico ni neurológico y a disminuir la tasa de mortalidad. ${ }^{1,2,8}$

En nuestro grupo la tasa de incidencia de R.N. de MBPN para el periodo fue de $1.35 \%$, valores similares a los descritos en la Unidad de Neonatología del Hospital Roberto del Rio, diferenciándose nuestro grupo por tener $22,9 \%$ de R.N. menores de 1.000 gramos en contraste con sólo $13 \%$ en dicha Lnidad. ${ }^{6}$

La tasa global de mortalidad en el grupo estudiado fue de $54,2 \%$, con $100 \%$ de mortalidad para los menores de I.O00 gramos y $40,7 \%$ para los R.N. con peso entre 1.001 y 1.500 gramos. 
Publicaciones extranjeras muestran que los cuidados intensivos han logrado disminuir estas cifras de mortalidad a niveles de 15 a $24 \%$ para niños de 1.001 a 1.500 gr y a $36 \%$ para niños menores de $1.000 \mathrm{gr}$.

Considerando que nuestro estudio fue hecho antes de la implementación perinatal, nuestras cifras no son comparables.

Esta nueva era para los R.N. de MBPN se caracteriza por un alcance clínico más positivo $y$ una tendencia a prestar máxima atención al control antes, durante y después del parto. ${ }^{1,2}$

Cada niño pequeño debe asistirse con monitoreo fetal cuidadoso, inhibicjón farmacológica de partos prematuros, prevención de partos traumáticos, resucitación rápida y oportuna por especialistas neonatólogos experimentados e intervención precoz en las alteraciones perinatales de la adaptación. ${ }^{1,2,9,10}$

Finalmente, queremos señalar, que sin la asistencia adecuada de los cuidados intensivos, se obtienen cifras como las señaladas en nuestro trabajo y que con la actual implementación perinatal a nuestro Servicio, esta realidad ya ha iniciado una variación significativa, lográndose bajar los índices de mortalidad sin aumentar ostensiblemente los indices de morbilidad.

\section{RESUMEN}

Se efectuó un eshudio retrospectivo de 35 fichas de niños nacidos entre el 15 de agosto de 1979 y el
30 de agosto de 1980 , que pesaron 1.500 gramos o menos al nacer.

Se analizaron la incidencia que tiene el peso, la E.G. y el Apgar sobre la mortalidad.

Se estableció que la incidencia de R.N. de MBPN es de $1.35 \%$ y la mortalidad, dentro de ese grupo, es de $54,2 \%$.

\section{REFERENCIAS}

1 fiexiew of books. The finte of the baby under $1.501 \mathrm{gr}$ ot birth, Ed. Lancet 1: (8166); 461-3 Mar. 1989.

${ }^{2}$ Hack, M. Curnent concept of the low birthweight infant evolution of changing cutloak. N. Engl. J. Med. 22: 301 (21) 1 162-5, Nov, 1979.

${ }^{3}$ Ferreira, $M$. Implementacion de la alención neonatal en Chile. Pediatra 19: 282, Dic. 1976.

4 Usher, $\boldsymbol{R}$. Judgement of fetel age; Clinical significance of gestional age and an objective method of its assesment. Pediatric Clin. N. An. 13: 835, 1966 .

${ }^{5}$ Amiel Tisson C. Neurological evalwation of Newbort Infints Arch. Dis. Chitd. 43: 89, 1968.

${ }^{6}$ Rizzardini, M. R.N. de M.B.P.N. Neomatologf 1: 6; 8 agosto 1980.

${ }^{7}$ Saransohn, $\mathrm{Ch}$. Care of the very sondl prematare infant. Pediatric Clinic. N. An. 24: 21-Aug. 197.

${ }^{8}$ Vohr, B. The preterm mall for gestational age infint, a two year follow up study. J. Obstet. Gymec. 133 (4) 425-31 Feb. 1979.

${ }^{9}$ Stectart. Improvement prognosis for v. L. b. w.'s infant. Pediatric's 54: 724-735: 1974.

${ }^{10}$ Clats, M., Faramoff, A. Care of the bigh Risk Neonates. 24:216. August 1877. 